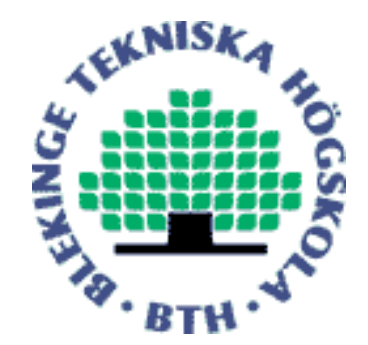

Copyright @ 2011 IEEE.

Citation for the published paper:

Title:

In press: Exact Performance of Two-Way AF Relaying in Nakagami-m Fading Environment

Author:

Jing Yang, Pingzhi Fan, Quang Trung Duong, Xianfu Lei

Journal:

IEEE Transaction on Wireless Communications

Year:

2011

Vol:

Issue:

99

Pagination:

1-8

URL/DOI to the paper:

10.1109/TWC.2011.101141

This material is posted here with permission of the IEEE. Such permission of the IEEE does not in any way imply IEEE endorsement of any of BTH's products or services Internal or personal use of this material is permitted. However, permission to reprint/republish this material for advertising or promotional purposes or for creating new collective works for resale or redistribution must be obtained from the IEEE by sending a blank email message to pubs-permissions@ieee.org.

By choosing to view this document, you agree to all provisions of the copyright laws protecting it. 


\title{
Exact Performance of Two-Way AF Relaying in Nakagami- $m$ Fading Environment
}

\author{
Jing Yang, Pingzhi Fan, Trung Q. Duong, and Xianfu Lei
}

\begin{abstract}
The performance of two-way amplify-and-forward (AF) relaying networks over independently but not necessarily identically distributed (i.n.i.d.) Nakagami- $m$ fading channels, with integer and integer plus one-half values of fading parameter $m$, is studied. Closed-form expressions for the cumulative distribution function (CDF), probability density function (PDF), and moment generating function (MGF) of the end-to-end signalto-noise ratio (SNR) are presented. Utilizing these results, we analyze the performance of two-way AF relaying system in terms of outage probability, average symbol error rate (SER), and average sum-rate. Simulations are performed to verify the correctness of our theoretical analysis.
\end{abstract}

Index Terms-Two-way relaying network, outage probability, average symbol error rate (SER), average sum-rate, Nakagami- $m$ fading.

\section{INTRODUCTION}

$\mathbf{I}$ $\mathrm{N}$ conventional cooperative communication networks, when all terminals are operated in half-duplex fashion, the transmission of one information symbol from the source terminal to the destination terminal occupies two channel uses [1]. This leads to a loss in spectral efficiency due to the pre-log factor one-half in corresponding to sum-rate expressions. To improve the spectral efficiency in half-duplex relaying, twoway relaying was proposed in [1], [2], where two source nodes simultaneously send their information to the relay node in the multiple-access (MA) phase and the relay node broadcasts the received signal to the two source nodes in the broadcast (BC) phase. In [1], Rankov and Wittneben have introduced the two-way relaying protocols, known as two-way amplifyand-forward (AF) relaying and two-way decode-and-forward (DF) relaying.

Recently, the performance of the conventional one-way cooperation system has been well investigated [3]-[9]. However,

Manuscript received June 28, 2010; revised October 17, 2010; accepted December 12, 2010. The associate editor coordinating the review of this paper and approving it for publication was S. Ghassemzadeh.

This work was supported by the National 863 High-Tech R \& D Program (No.2009AA01Z238), the National Science Foundation of China (No.60872013/60872014/60902023), the 111 Project (No.111-2-14), the SinoSwedish Cooperation Program (No.2008DFA12160), the Open Research Fund of State Key Laboratory of Integrated Services Networks (No.ISN11-04), and the Fundamental Research Funds for the Central Universities (No.2010XS21).

J. Yang, P. Fan, and X. Lei are with the Institute of Mobile Communications, Southwest Jiaotong University, Chengdu, Sichuan, 610031, P. R. of China. J. Yang and X. Lei are also with The State Key Laboratory of Integrated Services Networks, Xidian University, Xi'an, China (e-mail: jingyang95@gmail.com,p.fan@ieee.org,xflei81@yahoo.com.cn).

T. Q. Duong is with the Blekinge Institute of Technology, SE-371 79 Karlskrona, Sweden (e-mail: quang.trung.duong@bth.se).

Digital Object Identifier 10.1109/TWC.2011.101141. scanning the up-to-date open technical literature, the number of published works concerning performance analysis of twoway relaying system is relatively small. Han et al. presented an upper and lower bound for average sum-rate and an upper bound for pairwise error probability (PEP) in [10]. Ping and Ting investigated and compared the average rate performances in one-way and two-way AF relaying network [11]. Qiang et al. derived closed-form results for the outage probability in [12]. Later, Duong et al. obtained the exact closed-form expressions for outage probability, average symbol error rate (SER), and average sum-rate in [13].

While the aforementioned works assumed Rayleigh fading scenarios, to the best of our knowledge, the performance of two-way relaying system over generalized Nakagami- $m$ fading has not been investigated. It is well known that Nakagami- $m$ fading spans via the $m$ parameter a wide range of fading scenarios that are typical in realistic wireless relay applications, which includes the one-sided Gaussian distribution $(m=0.5)$ and Rayleigh fading $(m=1)$ as special cases. In practice, the radio links between the two source terminals and the relay may experience different fading scenario, which is a realistic assumption for a scenario where two source terminals are positioned different distance away from the relay node.

In this paper, we focus on the performance analysis of two-way AF relaying system in generally independently but not necessarily identically distributed (i.n.i.d.) Nakagami- $m$ fading channels. Specifically, we derive exact expressions for outage probability, average SER, and average sum-rate. We first present tractable forms for the statistics of the endto-end signal-to-noise ratio (SNR), e.g., cumulative distribution function (CDF), probability density function (PDF), and moment generating function (MGF). These new results are then applied to study the end-to-end performance of two-way relaying network, resulting in exact expressions for outage probability, average SER and average sum-rate. Note that for the case of Rayleigh fading channels, our general expressions for Nakagami- $m$ fading reduce to the previously published results presented in [13] as special cases. Analytical formulas are also verified by simulations.

\section{System AND ChanNel Model}

A simple two-way relaying system model operating over i.n.i.d. Nakagami- $m$ fading channels is illustrated in Fig. 1. Two source nodes $T_{1}$ and $T_{2}$ wish to exchange information with each other via an $\mathrm{AF}$ relay node $R$. Each transmission period is divided into two transmission phases: MA phase and BC phase. In the MA phase, $T_{1}$ and $T_{2}$ send simultaneously 


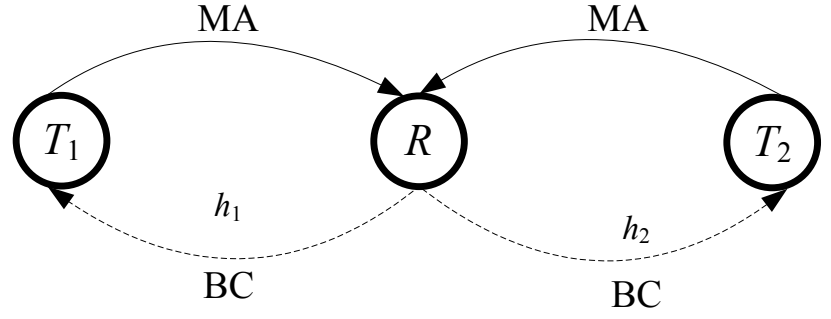

Fig. 1: System Model.

their information message to $R$. In the $\mathrm{BC}$ phase, $R$ normalizes the received signal based on its transmit power constraint and broadcasts to $T_{1}$ and $T_{2}$. As in [13], for simplicity of derivation, we assume equal transmit power (denoted as $P_{s}$ ) at $T_{1}, T_{2}$ and $R$ and equal variance (denoted as $N_{0}$ ) for the additive white Gaussian noise (AWGN) at the three terminals. Moreover, we presume that $T_{1}$ and $T_{2}$ have the perfect knowledge of the corresponding channel coefficients so that the self-interference can be fully eliminated. We also denote $h_{1}$ and $h_{2}$ as independent channel coefficients for $T_{1} \rightarrow R$ and $T_{2} \rightarrow R$ links, respectively, which are modeled as Nakagami$m$ random variables (RVs). Assume that the channels are reciprocal, so the channel gains of $T_{i} \rightarrow R$ and $R \rightarrow T_{i}$ (i $=1,2)$ links are identical [1]. Hence, the instantaneous SNR of terminal $T_{i}$ in the BC phase, $\gamma_{T_{i}}$, can be given by [1], [10], [13]

$$
\gamma_{T_{i}}=\frac{\gamma_{i} \gamma_{j}}{2 \gamma_{i}+\gamma_{j}}
$$

where $\gamma_{i}=P_{s}\left|h_{i}\right|^{2} / N_{0}$ and $\gamma_{j}=P_{s}\left|h_{j}\right|^{2} / N_{0}(i, j=1,2$ and $i \neq j$ ) are the instantaneous SNRs for $T_{i} \rightarrow R$ and $R \rightarrow T_{j}$ links, respectively. Note that $\gamma_{0}=P_{s} / N_{0}$ is the average transmit SNR and $\Omega_{i}=\mathbb{E}\left\{h_{i}^{2}\right\}, \Omega_{j}=\mathbb{E}\left\{h_{j}^{2}\right\}$ are the respective variances of $h_{i}$ and $h_{j}$, where $\mathbb{E}\{\cdot\}$ denotes the statistical average operator. Since $h_{i}$ is modeled as Nakagami$m \mathrm{RV}$, the instantaneous SNR, $\gamma_{i}$ is a gamma distributed RV with PDF given by

$$
f_{\gamma_{i}}(\gamma)=\frac{m_{i}^{m_{i}}}{\bar{\gamma}_{i}^{m_{i}} \Gamma\left(m_{i}\right)} \gamma^{m_{i}-1} e^{-\frac{m_{i \gamma} \gamma}{\bar{\gamma}_{i}}}
$$

where $\Gamma(\cdot)$ is the gamma function [14, Eq. (8.310.1)], $m_{i}$ is the Nakagami fading parameter and $\bar{\gamma}_{i}=\Omega_{i} \gamma_{0}$ is the average SNR of the $T_{i} \rightarrow R$ link. Hence, its CDF can be written as

$$
F_{\gamma_{i}}(\gamma)=1-\frac{\Gamma\left(m_{i},\left(m_{i} / \bar{\gamma}_{i}\right) \gamma\right)}{\Gamma\left(m_{i}\right)}
$$

where $\Gamma(\cdot, \cdot)$ is the incomplete gamma function defined in [14, Eq. (8.350.2)].

\section{Performance Analysis of Two-Way AF RELAYING NETWORK}

We first present the CDF and PDF of $\gamma_{T_{i}}$, which will be used for the derivation of the performance metrics.
Theorem 1: When $m$ is integer, the $\mathrm{CDF}$ of $\gamma_{T_{i}}$ is given by

$$
\begin{gathered}
F_{\gamma_{T_{i}}}(\gamma)=1-\frac{2 m_{i}^{m_{i}} e^{-b_{i} \gamma}\left(m_{j}-1\right) !}{\bar{\gamma}_{i}^{m_{i}} \Gamma\left(m_{i}\right) \Gamma\left(m_{j}\right)} \sum_{p_{i}=0}^{m_{i}-1} \sum_{p_{j}=0}^{m_{j}-1} \sum_{k=0}^{p_{j}} \frac{1}{p_{j} !}\left(\begin{array}{c}
p_{j} \\
k
\end{array}\right) \\
\times\left(\begin{array}{c}
m_{i}-1 \\
p_{i}
\end{array}\right)\left(\frac{m_{i}}{\bar{\gamma}_{i}}\right)^{-\frac{\sigma_{i}+1}{2}}\left(\frac{2 m_{j}}{\bar{\gamma}_{j}}\right)^{p_{j}+\frac{\sigma_{i}+1}{2}} \\
\times \gamma^{\delta_{i}} K_{\sigma_{i}+1}(a \gamma),
\end{gathered}
$$

where $\delta_{i}=m_{i}+p_{j}, \sigma_{i}=p_{i}-k, b_{i}=m_{i} / \bar{\gamma}_{i}+2 m_{j} / \bar{\gamma}_{j}$, $a=2 \sqrt{2 m_{i} m_{j} /\left(\bar{\gamma}_{i} \bar{\gamma}_{j}\right)}$ and $K_{v}(\cdot)$ is the $v$-th order modified Bessel function of the second kind [14, Eq. (8.432.6)].

Proof: See Appendix A.

Theorem 2: When $m$ is integer plus one half, the CDF of $\gamma_{T_{i}}$ is given by

$$
\begin{aligned}
F_{\gamma_{T_{i}}}(\gamma)=1- & \frac{\sqrt{\pi}}{\Gamma\left(m_{i}\right) \Gamma\left(m_{j}\right)}\left(\frac{m_{i}}{\bar{\gamma}_{i}}\right)^{\frac{m_{i}}{2}-\frac{1}{4}}\left(\frac{2 m_{j}}{\bar{\gamma}_{j}}\right)^{\frac{m_{j}}{2}-\frac{1}{4}} \\
& \times \sum_{p_{i}=0}^{m_{i}-\frac{1}{2}} \sum_{p_{j}=0}^{m_{j}-\frac{1}{2}} \frac{\gamma^{\frac{\alpha}{2}} e^{-c_{i}^{2} \gamma} H_{\alpha-1}\left(c_{i} \sqrt{\gamma}\right)}{2^{\alpha-1}} E,
\end{aligned}
$$

where

$$
\begin{aligned}
& \alpha=\sum_{l=1}^{2}\left(m_{l}-p_{l}\right)-1, \\
& c_{i}=\sqrt{\frac{m_{i}}{\bar{\gamma}_{i}}}+\sqrt{\frac{2 m_{j}}{\bar{\gamma}_{j}}},
\end{aligned}
$$$$
E=\frac{\left(m_{i}+p_{i}-\frac{1}{2}\right) !\left(m_{j}+p_{j}-\frac{1}{2}\right) !\left(\frac{m_{i}}{\bar{\gamma}_{i}}\right)^{-\frac{p_{i}}{2}}\left(\frac{2 m_{j}}{\bar{\gamma}_{j}}\right)^{-\frac{p_{j}}{2}}}{4^{p_{i}+p_{j}} p_{i} ! p_{j} !\left(m_{i}-p_{i}-\frac{1}{2}\right) !\left(m_{j}-p_{j}-\frac{1}{2}\right) !},
$$

and

$$
H_{n}(x)=n ! \sum_{k=0}^{\left\lfloor\frac{n}{2}\right\rfloor} \frac{(-1)^{k}(2 x)^{n-2 k}}{k !(n-2 k) !},
$$

is the Hermite polynomials [14, Eq.(8.951].

Proof: See Appendix B.

Corollary 1: When $m$ is integer plus one half, the PDF of $\gamma_{T_{i}}$ is given by

$$
\begin{aligned}
f_{\gamma_{T_{i}}}(\gamma)= & \frac{\sqrt{\pi}}{\Gamma\left(m_{i}\right) \Gamma\left(m_{j}\right)}\left(\frac{m_{i}}{\bar{\gamma}_{i}}\right)^{\frac{m_{i}}{2}-\frac{1}{4}}\left(\frac{2 m_{j}}{\bar{\gamma}_{j}}\right)^{\frac{m_{j}}{2}-\frac{1}{4}} \\
& \times \sum_{p_{i}=0}^{m_{i}-\frac{1}{2}} \sum_{p_{j}=0}^{m_{j}-\frac{1}{2}} \frac{\gamma^{\frac{\alpha}{2}-1} e^{-c_{i}^{2} \gamma} H_{\alpha+1}\left(c_{i} \sqrt{\gamma}\right)}{2^{\alpha+1}} E .
\end{aligned}
$$

Proof: See Appendix C.

\section{A. Outage Probability}

The outage probability $P_{o u t, T_{i}}\left(\gamma_{t h}\right)$ at $T_{i}$ is defined as the probability that the instantaneous SNR falls below a given threshold $\gamma_{t h}$,

$$
P_{\text {out }, T_{i}}\left(\gamma_{t h}\right)=\operatorname{Pr}\left(\gamma_{T_{i}}<\gamma_{t h}\right)=F_{\gamma_{T_{i}}}\left(\gamma_{t h}\right),
$$

where $F_{\gamma_{T_{i}}}\left(\gamma_{t h}\right)$ is the CDF of the instantaneous SNR at terminal $T_{i}$, evaluated at $\gamma=\gamma_{t h}$. 
It is clear now that by using (4), (5), and (8), the outage probability of two-way AF relaying network over Nakagami$m$ fading channels with integer and integer plus one half values of $m$ at terminal $T_{i}$ can be easily expressed in closed form.

For the case of Rayleigh fading channel, i.e., $m=1$, we can see that our result particularizes to [13, Eq. (16)].

When $m=0.5$, the Nakagami distribution corresponds to a one-sided Gaussian distribution, which is an important special case as it represents a worst case fading scenario. Especially, the obtained result of outage probability for $m=0.5$ will have a significant practical usefulness in wireless relaying systems when quality of service requirements mandate designing for worst-case fading.

\section{B. Average Symbol Error Rate}

In order to obtain the average SER for a large variety of modulation schemes, the MGF based approach [15] will be used in this paper. Based on the fact that $\Phi_{\gamma_{T_{i}}}(s)=$ $\mathbb{E}_{\gamma_{T_{i}}}\{\exp (-s \gamma)\}$, to evaluate the average SER, the PDF $f_{\gamma_{T_{i}}}(\gamma)$ and the MGF $\Phi_{\gamma_{T_{i}}}(s)$ will be firstly obtained in the following.

1) Integer values of $m$ : In this case, $f_{\gamma_{T_{i}}}(\gamma)$ can be found by taking the first derivative of (4) with respect to $\gamma$, yielding

$$
\begin{aligned}
f_{\gamma_{T_{i}}}(\gamma)=-\frac{2 m_{i}^{m_{i}}\left(m_{j}-1\right) !}{\bar{\gamma}_{i}^{m_{i}} \Gamma\left(m_{i}\right) \Gamma\left(m_{j}\right)} \sum_{p_{i}=0}^{m_{i}-1} \sum_{p_{j}=0}^{m_{j}-1} \sum_{k=0}^{p_{j}} \frac{1}{p_{j} !}\left(\begin{array}{c}
p_{j} \\
k
\end{array}\right) \\
\times\left(\begin{array}{c}
m_{i}-1 \\
p_{i}
\end{array}\right)\left(\frac{m_{i}}{\bar{\gamma}_{i}}\right)^{-\frac{\sigma_{i}+1}{2}}\left(\frac{2 m_{j}}{\bar{\gamma}_{j}}\right)^{\frac{2 p_{j}+\sigma_{i}+1}{2}} \\
\quad \times\left[L_{1}^{\prime}(\gamma) L_{2}(\gamma)+L_{1}(\gamma) L_{2}^{\prime}(\gamma)\right], \quad(9)
\end{aligned}
$$

Recalling the relationship between $\Phi_{\gamma_{T_{i}}}(s)$ and $f_{\gamma_{T_{i}}}(\gamma)$, $\Phi_{\gamma_{T_{i}}}(s)$ can be determined by

$$
\begin{aligned}
\Phi_{\gamma_{T_{i}}}(s)= & -\frac{2 m_{i}^{m_{i}}\left(m_{j}-1\right) !}{\bar{\gamma}_{i}^{m_{i}} \Gamma\left(m_{i}\right) \Gamma\left(m_{j}\right)} \sum_{p_{i}=0}^{m_{i}-1} \sum_{p_{j}=0}^{m_{j}-1} \sum_{k=0}^{p_{j}} \frac{1}{p_{j} !}\left(\begin{array}{c}
p_{j} \\
k
\end{array}\right) \\
& \times\left(\begin{array}{c}
m_{i}-1 \\
p_{i}
\end{array}\right)\left(\frac{m_{i}}{\bar{\gamma}_{i}}\right)^{-\frac{\sigma_{i}+1}{2}}\left(\frac{2 m_{j}}{\bar{\gamma}_{j}}\right)^{\frac{2 p_{j}+\sigma_{i}+1}{2}} \\
\times & \int_{0}^{\infty}\left[L_{1}^{\prime}(\gamma) L_{2}(\gamma)+L_{1}(\gamma) L_{2}^{\prime}(\gamma)\right] e^{-s \gamma} d \gamma, \quad(10)
\end{aligned}
$$

where $L_{1}(\gamma)=e^{-b_{i} \gamma}, L_{2}(\gamma)=\gamma^{\delta_{i}} K_{\sigma_{i}+1}(a \gamma)$ and $L_{i}^{\prime}(\gamma)$ with $i=1,2$ is the first derivative of $L_{i}(\gamma)$.

From [14, Eq. (8.486.12)] and [14, Eq. (6.621.3)], (10) can be calculated in closed-form as

$$
\begin{aligned}
\Phi_{\gamma_{T_{i}}}(s)=-\frac{2 m_{i}^{m_{i}}\left(m_{j}-1\right) !}{\bar{\gamma}_{i}^{m_{i}}} \Gamma\left(m_{i}\right) \Gamma\left(m_{j}\right) & \sum_{p_{i}=0}^{m_{i}-1} \sum_{p_{j}=0}^{m_{j}-1} \sum_{k=0}^{p_{j}} \frac{1}{p_{j} !}\left(\begin{array}{c}
p_{j} \\
k
\end{array}\right) \\
\times & \left(\begin{array}{c}
m_{i}-1 \\
p_{i}
\end{array}\right)\left(\frac{m_{i}}{\bar{\gamma}_{i}}\right)^{-\frac{\sigma_{i}+1}{2}}\left(\frac{2 m_{j}}{\bar{\gamma}_{j}}\right)^{\frac{2 p_{j}+\sigma_{i}+1}{2}} \\
& \times\left[b_{i} s_{1}+\left(\delta_{i}-\left(\sigma_{i}+1\right)\right) s_{2}+a s_{3}\right], \quad(11)
\end{aligned}
$$

where

$$
\begin{aligned}
s_{1}= & -\frac{\sqrt{\pi}(2 a)^{\sigma_{i}+1} \Gamma\left(\delta_{i}+\sigma_{i}+2\right) \Gamma\left(\delta_{i}-\sigma_{i}\right)}{\left(b_{i}+s+a\right)^{\delta_{i}+\sigma_{i}+2} \Gamma\left(\delta_{i}+\frac{3}{2}\right)} \\
& \times{ }_{2} F_{1}\left(\delta_{i}+\sigma_{i}+2, \sigma_{i}+\frac{3}{2} ; \delta_{i}+\frac{3}{2} ; \frac{b_{i}+s-a}{b_{i}+s+a}\right), \\
s_{2}= & \frac{\sqrt{\pi}(2 a)^{\sigma_{i}+1} \Gamma\left(\delta_{i}+\sigma_{i}+1\right) \Gamma\left(\delta_{i}-\left(\sigma_{i}+1\right)\right)}{\left(b_{i}+s+a\right)^{\delta_{i}+\sigma_{i}+1} \Gamma\left(\delta_{i}+\frac{1}{2}\right)} \\
& \times{ }_{2} F_{1}\left(\delta_{i}+\sigma_{i}+1, \sigma_{i}+\frac{3}{2} ; \delta_{i}+\frac{1}{2} ; \frac{b_{i}+s-a}{b_{i}+s+a}\right),
\end{aligned}
$$

$$
\begin{aligned}
s_{3}=- & \frac{\sqrt{\pi}(2 a)^{\sigma_{i}} \Gamma\left(\delta_{i}+\sigma_{i}+1\right) \Gamma\left(\delta_{i}-\sigma_{i}+1\right)}{\left(b_{i}+s+a\right)^{\delta_{i}+\sigma_{i}+1} \Gamma\left(\delta_{i}+\frac{3}{2}\right)} \\
& \times{ }_{2} F_{1}\left(\delta_{i}+\sigma_{i}+1, \sigma_{i}+\frac{1}{2} ; \delta_{i}+\frac{3}{2} ; \frac{b_{i}+s-a}{b_{i}+s+a}\right),
\end{aligned}
$$

and ${ }_{p} F_{q}\left(a_{1}, \ldots, a_{p} ; b_{1}, \ldots, b_{q} ; x\right)$ is the generalized hypergeometric function [14, Eq.(9.14.1)].

For $m=1$, (11) exactly agrees with [13, Eq. (9)].

2) Integer plus one-half values of $m$ : In this case, with the help of (7), $\Phi_{\gamma_{T_{i}}}(s)$ can be determined by

$$
\begin{aligned}
\Phi_{\gamma_{T_{i}}}(s)= & \frac{\sqrt{\pi}}{\Gamma\left(m_{i}\right) \Gamma\left(m_{j}\right)}\left(\frac{m_{i}}{\bar{\gamma}_{i}}\right)^{\frac{m_{i}}{2}-\frac{1}{4}}\left(\frac{2 m_{j}}{\bar{\gamma}_{j}}\right)^{\frac{m_{j}}{2}-\frac{1}{4}} \\
& \times \sum_{p_{i}=0}^{m_{i}-\frac{1}{2}} \sum_{p_{j}=0}^{m_{j}-\frac{1}{2}\left\lfloor\frac{\alpha+1}{2}\right\rfloor} \sum_{k=0}^{\infty} \frac{(-1)^{k} E \times(\alpha+1) !\left(2 c_{i}\right)^{\alpha+1-2 k}}{2^{\alpha+1} k !(\alpha+1-2 k) !} \\
& \times \int_{0}^{\infty} e^{-\left(c_{i}^{2}+s\right) \gamma} \gamma^{\frac{2 \alpha-2 k-1}{2}} d \gamma
\end{aligned}
$$

From [14, Eq. (3.326.2)], (12) can be calculated as

$$
\begin{aligned}
\Phi_{\gamma_{T_{i}}}(s)= & \frac{\sqrt{\pi}}{\Gamma\left(m_{i}\right) \Gamma\left(m_{j}\right)}\left(\frac{m_{i}}{\bar{\gamma}_{i}}\right)^{\frac{m_{i}}{2}-\frac{1}{4}}\left(\frac{2 m_{j}}{\bar{\gamma}_{j}}\right)^{\frac{m_{j}}{2}-\frac{1}{4}} \\
& \times \sum_{p_{i}=0}^{m_{i}-\frac{1}{2}} \sum_{p_{j}=0}^{m_{j}-\frac{1}{2}\left\lfloor\frac{\alpha+1}{2}\right\rfloor} \sum_{k=0}^{\frac{(-1)^{k}(\alpha+1) !\left(2 c_{i}\right)^{\alpha-2 k+1}}{2^{\alpha+1} k !(\alpha-2 k+1) !}} \\
& \times\left(c_{i}^{2}+s\right)^{-u} \Gamma(u) E, \quad(13)
\end{aligned}
$$

where $u=(2 \alpha-2 k+1) / 2$.

For $m=0.5$, one can obtain the MGF of $\gamma_{T_{i}}$ from (13) in one-sided Gaussian distribution fading channel.

The derived MGF, $\Phi_{\gamma_{T_{i}}}(s)$ in (11) and (13), can be used to evaluate the average SER. For instance, the average SER for M-PSK at $T_{i}$ can be expressed as [15, Eq. (9.15)]

$$
P_{s}^{T_{i}}=\frac{1}{\pi} \int_{0}^{(\pi-\pi / M)} \Phi_{\gamma_{T_{i}}}\left(\frac{g}{\sin ^{2} \theta}\right) d \theta
$$

where $g=\sin ^{2}(\pi / M)$.

The exact SER expression given in (14) readily enable us to evaluate the SER performance by utilizing some efficient numerical integration techniques. 


\section{Average Sum-Rate}

The average sum-rate of the two-way relaying networks is defined as the summation of average rate of $T_{1}$ and $T_{2}$ and can be shown as [13]

$$
R_{\text {sum }}=\sum_{i=1}^{2} \mathbb{E}_{\gamma_{T_{i}}}\left\{\frac{1}{2} \log _{2}\left(1+\gamma_{T_{i}}\right)\right\}=\sum_{i=1}^{2} R_{\text {sum }, T_{i}} .
$$

1) Integer values of $m$ : Based on (9), we can rewrite the summand of (15) as

$$
\begin{aligned}
& R_{\text {sum }, T_{i}}=-\frac{1}{2 \ln 2} \frac{2 m_{i}^{m_{i}}\left(m_{j}-1\right) !}{\bar{\gamma}_{i}^{m_{i}} \Gamma\left(m_{i}\right) \Gamma\left(m_{j}\right)} \sum_{p_{i}=0}^{m_{i}-1} \sum_{p_{j}=0}^{m_{j}-1} \sum_{k=0}^{p_{j}} \frac{1}{p_{j} !}\left(\begin{array}{c}
p_{j} \\
k
\end{array}\right) \\
& \times\left(\begin{array}{c}
m_{i}-1 \\
p_{i}
\end{array}\right)\left(\frac{m_{i}}{\bar{\gamma}_{i}}\right)^{-\frac{\sigma_{i}+1}{2}}\left(\frac{2 m_{j}}{\bar{\gamma}_{j}}\right)^{\frac{2 p_{j}+\sigma_{i}+1}{2}} \\
& \times \int_{0}^{\infty}\left[L_{1}^{\prime}(\gamma) L_{2}(\gamma)+L_{1}(\gamma) L_{2}^{\prime}(\gamma)\right] \ln (1+\gamma) d \gamma .
\end{aligned}
$$

After some elementary manipulation, (16) can be expressed as

$$
\begin{aligned}
R_{\mathrm{sum}, T_{i}}=-\frac{1}{2 \ln 2} \frac{2 m_{i}^{m_{i}}\left(m_{j}-1\right) !}{\bar{\gamma}_{i}^{m_{i}} \Gamma\left(m_{i}\right) \Gamma\left(m_{j}\right)} \sum_{p_{i}=0}^{m_{i}-1} \sum_{p_{j}=0}^{m_{j}-1} \sum_{k=0}^{p_{j}} \frac{1}{p_{j} !}\left(\begin{array}{c}
p_{j} \\
k
\end{array}\right) \\
\times\left(\begin{array}{c}
m_{i}-1 \\
p_{i}
\end{array}\right)\left(\frac{m_{i}}{\bar{\gamma}_{i}}\right)^{-\frac{\sigma_{i}+1}{2}}\left(\frac{2 m_{j}}{\bar{\gamma}_{j}}\right)^{p_{j}+\frac{\sigma_{i}+1}{2}} \\
\times\left(t_{1}+t_{2}+t_{3}\right),(17)
\end{aligned}
$$

where

$$
\begin{aligned}
& t_{1}=-b_{i} \int_{0}^{\infty} \gamma^{\delta_{i}} e^{-b_{i} \gamma} \ln (1+\gamma) K_{\sigma_{i}+1}(a \gamma) d \gamma, \\
& t_{2}=\left(\delta_{i}-\sigma_{i}-1\right) \\
& \times \int_{0}^{\infty} \gamma^{\delta_{i}-1} e^{-b_{i} \gamma} \ln (1+\gamma) K_{\sigma_{i}+1}(a \gamma) d \gamma, \\
& t_{3}=-a \int_{0}^{\infty} \gamma^{\delta_{i}} e^{-b_{i} \gamma} \ln (1+\gamma) K_{\sigma_{i}}(a \gamma) d \gamma .
\end{aligned}
$$

In order to evaluate $R_{\mathrm{sum}, T_{i}}$, we will utilize the result of $J=\int_{0}^{\infty} x^{m} e^{-p x} \ln (1+x) K_{n}(q x) d x$, which is derived in Appendix D. Applying (37) into (17) yields the closed-form expression of the summand $R_{\mathrm{sum}, T_{i}}$ which helps us to obtain the average sum-rate $R_{\text {sum }}$ as follows:

$$
\begin{aligned}
& R_{\mathrm{sum}}=- \frac{1}{2 \ln 2} \sum_{i=1}^{2} \sum_{p_{i}=0}^{m_{i}-1} \sum_{p_{j}=0}^{m_{j}-1} \sum_{k=0}^{p_{j}} \frac{2 m_{i}^{m_{i}}\left(m_{j}-1\right) !}{\bar{\gamma}_{i}^{m_{i}} \Gamma\left(m_{i}\right) \Gamma\left(m_{j}\right)} \frac{1}{p_{j} !} \\
& \times\left(\begin{array}{c}
p_{j} \\
k
\end{array}\right)\left(\begin{array}{c}
m_{i}-1 \\
p_{i}
\end{array}\right)\left(\frac{m_{i}}{\bar{\gamma}_{i}}\right)^{-\frac{\sigma_{i}+1}{2}}\left(\frac{2 m_{j}}{\bar{\gamma}_{j}}\right)^{p_{j}+\frac{\sigma_{i}+1}{2}} \\
& \times\left(R_{T_{i}, A}+R_{T_{i}, B}+R_{T_{i}, C}\right),
\end{aligned}
$$

where

$$
\begin{aligned}
& R_{T_{i}, A}=-b_{i} \sqrt{\pi}\left(b_{i}-a\right)^{-\delta_{i}-1} \times H_{\mathrm{temp} 1}, \\
& R_{T_{i}, B}=\left(\delta_{i}-\sigma_{i}-1\right) \sqrt{\pi}\left(b_{i}-a\right)^{-\delta_{i}} \times H_{\mathrm{temp} 1}, \\
& R_{T_{i}, C}=-a \sqrt{\pi}\left(b_{i}-a\right)^{-\delta_{i}-1} \times H_{\mathrm{temp} 2},
\end{aligned}
$$

$$
\begin{aligned}
& H_{\mathrm{temp} 1}= \\
& H_{1,[2: 1], 0,[2: 2]}^{1,2,0,1,2} \\
& {\left[\begin{array}{c|c}
\frac{1}{b_{i}-a} & (2,1) \\
& (1,1),(1,1) ;\left(\frac{1}{2}, 1\right) \\
\frac{2 a}{b_{i}-a} & (1,1),(0,1) ;\left(\xi_{i}, 1\right),\left(-\xi_{i}, 1\right)
\end{array}\right]}
\end{aligned}
$$

$H_{\text {temp2 }}=$

$$
H_{1,[2: 1], 0,[2: 2]}^{1,2,0,1,2}\left[\begin{array}{c|c}
\frac{1}{b_{i}-a} & (2,1) \\
& (1,1),(1,1) ;\left(\frac{1}{2}, 1\right) \\
-- & 2 a \\
\frac{2 a}{b_{i}-a} & (1,1),(0,1) ;\left(\sigma_{i}, 1\right),\left(-\sigma_{i}, 1\right)
\end{array}\right],
$$

and $\xi_{i}=\sigma_{i}+1$.

The average sum-rate in two-way AF relaying network over Nakagami- $m$ fading channels with integer values of $m$ can be evaluated by using (18). As can be seen from (18), the fading parameter $m$ affects the average sum-rate performance.

When $m=1$, our obtained average sum-rate reduces to [13, Eq. (15)].

2) Integer plus one-half values of $m$ : Based on (7), we can rewrite the summand of (15) as

$$
\begin{aligned}
R_{\mathrm{sum}, T_{i}}=\frac{1}{2 \ln 2} & \sum_{p_{i}=0}^{m_{i}-\frac{1}{2}} \sum_{p_{j}=0}^{m_{j}-\frac{1}{2}\left\lfloor\frac{\alpha+1}{2}\right\rfloor} \frac{\sqrt{\pi}}{\Gamma\left(m_{i}\right) \Gamma\left(m_{j}\right)} \\
\times\left(\frac{m_{i}}{\bar{\gamma}_{i}}\right)^{\frac{m_{i}}{2}-\frac{1}{4}} & \left(\frac{2 m_{j}}{\bar{\gamma}_{j}}\right)^{\frac{m_{j}}{2}-\frac{1}{4}} \frac{(-1)^{k}(\alpha+1) !\left(2 c_{i}\right)^{\alpha+1-2 k} E}{2^{\alpha+1} k !(\alpha+1-2 k) !} \\
& \times \int_{0}^{\infty} e^{-c_{i}^{2} \gamma} \gamma^{\frac{2 \alpha-2 k-1}{2}} \ln (1+\gamma) d \gamma . \quad \text { (19) }
\end{aligned}
$$

Finally, using [14, Eq.(4.352.1)] yields the closed-form expression of the summand $R_{\mathrm{sum}, T_{i}}$ which helps us to obtain the average sum-rate as follows:

$$
\begin{aligned}
& R_{\text {sum }}=\frac{1}{2 \ln 2} \sum_{i=1}^{2} \sum_{p_{i}=0}^{m_{i}-\frac{1}{2}} \sum_{p_{j}=0}^{m_{j}-\frac{1}{2}\left\lfloor\frac{\alpha+1}{2}\right\rfloor} \frac{\sqrt{\pi}}{\Gamma\left(m_{i}\right) \Gamma\left(m_{j}\right)} \\
& \times\left(\frac{m_{i}}{\bar{\gamma}_{i}}\right)^{\frac{m_{i}}{2}-\frac{1}{4}}\left(\frac{2 m_{j}}{\bar{\gamma}_{j}}\right)^{\frac{m_{j}}{2}-\frac{1}{4}} \frac{(-1)^{k}(\alpha+1) !\left(2 c_{i}\right)^{\alpha+1-2 k} E}{2^{\alpha+1} k !(\alpha+1-2 k) !} \\
& \times R_{T_{i}, D}, \quad(20)
\end{aligned}
$$

where

$$
\begin{aligned}
R_{T_{i}, D} & =\frac{{ }_{1} F_{1}\left(u ; u+1 ; c_{i}^{2}\right)}{u \sin (u \pi)} \pi-c_{i}^{-2 u} \Gamma(u) \\
& \times\left[2 \ln c_{i}-\psi(u)+\frac{{ }_{2} F_{2}\left(1,1 ; 2,2-u ; c_{i}^{2}\right)}{1-u} c_{i}^{2}\right],
\end{aligned}
$$

and $\psi(\cdot)$ denotes the Euler psi function [14, Eq.(8.360.1)], $j=1,2$ and $j \neq i$.

Again, it can also be seen from (20), average sum-rate performance depends on the fading severity parameter $m$. When $m=0.5$, our result given in (20) becomes the average sum-rate of two-way AF relaying network over one-sided Gaussian distribution fading channel. 


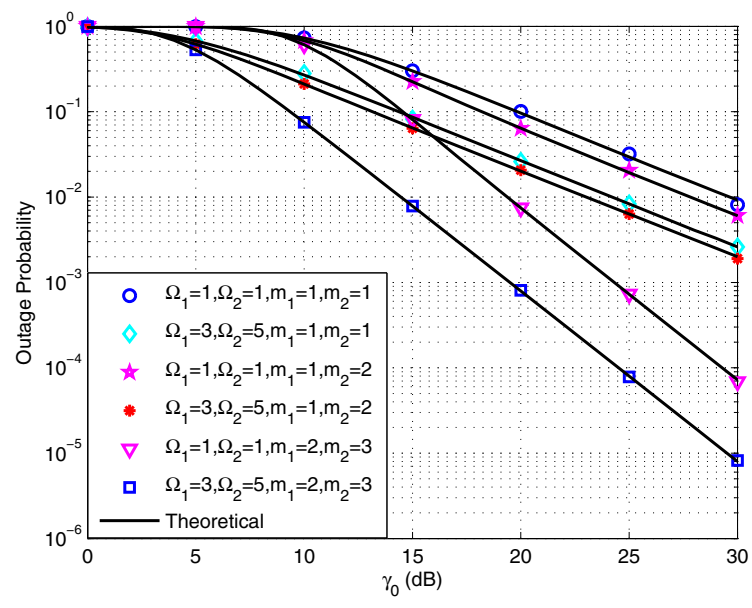

Fig. 2: Outage probability for the two-way AF relaying at terminal $T_{2}$ with integer values of $m$.

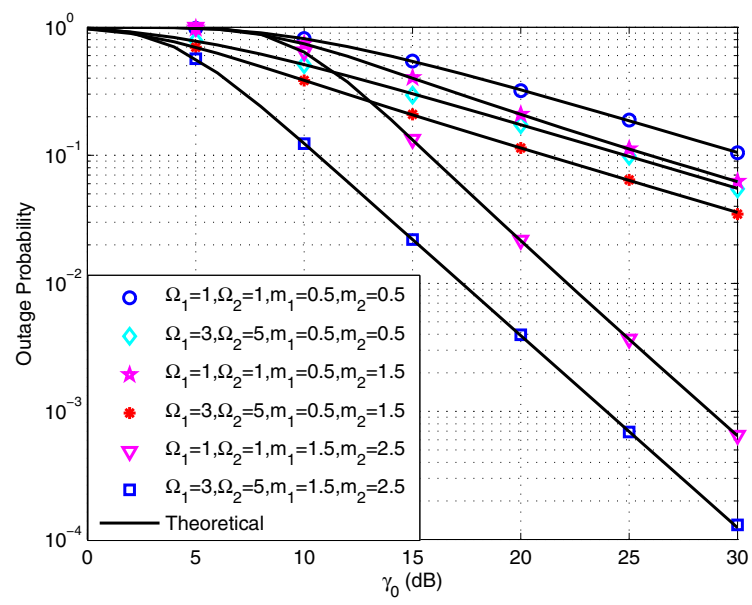

Fig. 3: Outage probability for the two-way AF relaying at terminal $T_{2}$ with integer plus one-half values of $m$.

\section{Numerical Results}

In this section, simulation is provided to demonstrate the validity and usefulness of our analytical expressions. For numerical results, the special function in the formulas can be easily evaluated by using common mathematical software packages such as Mathematica or Maple. In order to compare the results in [13], we also consider two examples: 1) i.i.d. case (e.g., $\Omega_{1}=\Omega_{2}=1$ ) and 2) i.n.i.d. case (e.g., $\Omega_{1}=3, \Omega_{2}=5$ ). Different fading condition, such as different fading parameters, is considered. The Rayleigh faded performance is also plotted. The solid lines in Figs. 2-7 show the theoretical results for outage probability, average SER and average sum-rate, which are obtained by the analytical expressions in this paper, while the other lines represent the simulation results.

Fig. 2 and Fig. 3 draw respectively the outage probability versus $\gamma_{0}$ with $\gamma_{t h}=3$ at terminal $T_{2}$ when fading parameter is integer and integer plus one-half. As can be clearly seen from both figures, analytical and simulated outage probability curves match excellently. As expected, improved fading severity conditions or fading conditions in two links

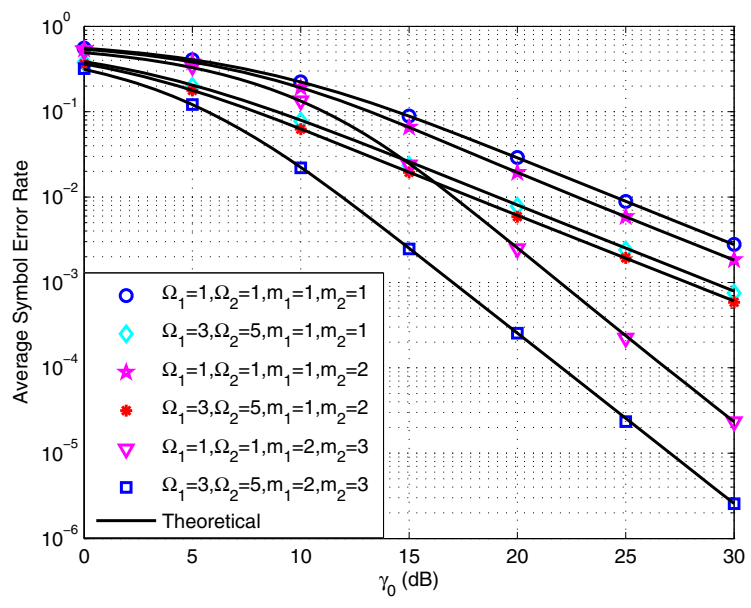

Fig. 4: Average SER for the two-way AF relaying at terminal $T_{2}$ with integer values of $m$.

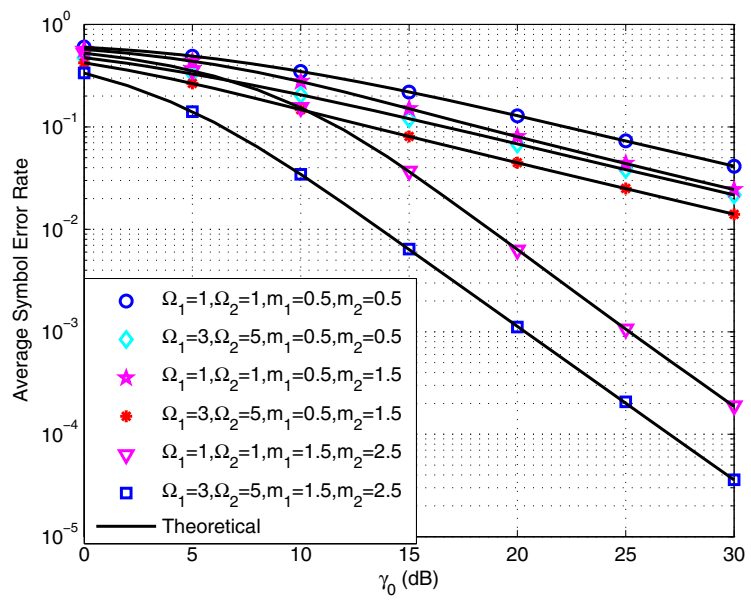

Fig. 5: Average SER for the two-way AF relaying at terminal $T_{2}$ with integer plus one-half values of $m$.

can significantly lower outage probability. For example, when an outage probability equals to $10^{-2}$, when fading parameters change from $m_{1}=1, m_{2}=1$ to $m_{1}=2, m_{2}=3$, an SNR gain of $15 \mathrm{~dB}$ can be achieved (for i.i.d case, see Fig. 2).

Fig. 4 and Fig. 5 present the average SER results for QPSK modulation at terminal $T_{2}$, respectively for fading parameter being integer and integer plus one-half. We see that the simulation results also closely match with the analytical ones. Results show that the average SER performance can be significantly improved for various fading parameter. For example, when average SER equals to $10^{-1}$, when fading parameters change from $m_{1}=0.5, m_{2}=0.5$ to $m_{1}=1.5$, $m_{2}=2.5$, an SNR gain of $10 \mathrm{~dB}$ can be achieved (for i.n.i.d case, see Fig. 5).

Fig. 6 and Fig. 7 show the average sum-rate performance. It can also be seen that the simulation results closely match with the analytical ones. As expected, improved fading parameter in two links can increase average sum-rate. For instance, when average sum-rate equals to 2 , when fading parameter from $m_{1}=0.5, m_{2}=0.5$ to $m_{1}=1.5, m_{2}=2.5$, a SNR gain of 


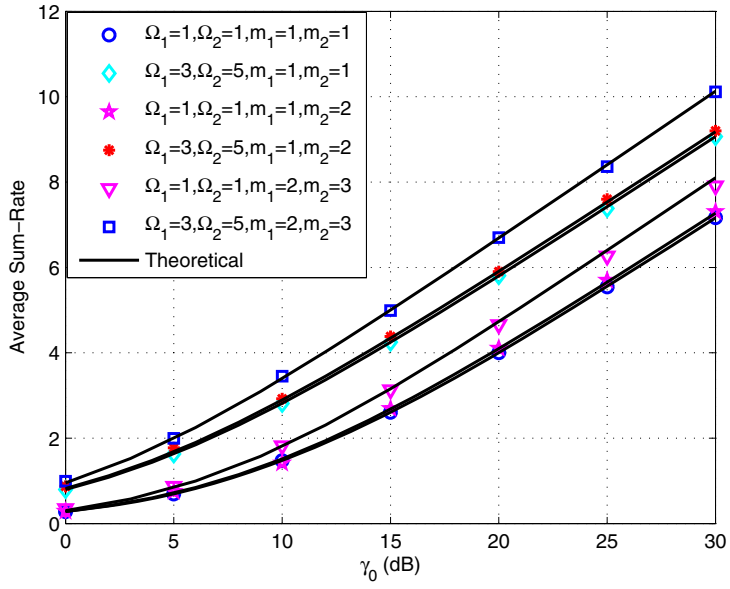

Fig. 6: Average sum-rate for the two-way AF relaying with integer values of $m$.

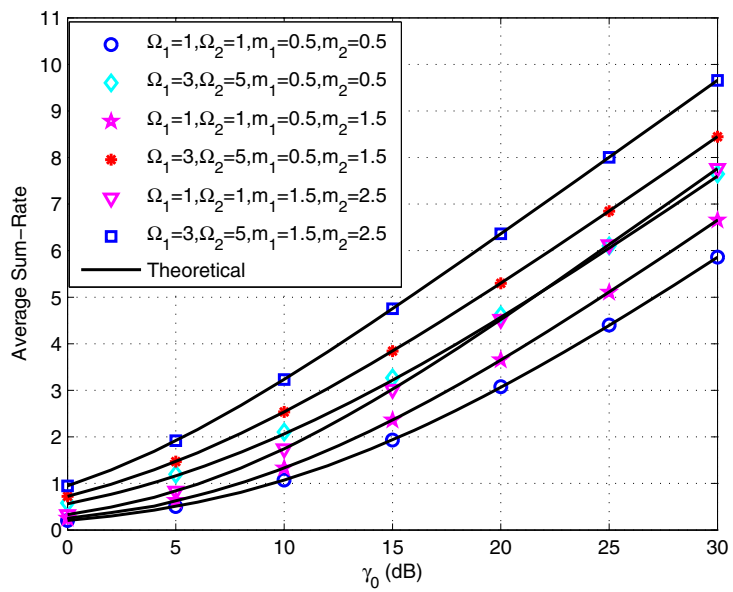

Fig. 7: Average sum-rate for the two-way AF relaying with integer plus one-half values of $m$.

$5 \mathrm{~dB}$ can be achieved (for i.n.i.d case, see Fig. 7).

Note that for Rayleigh fading channels, all results confirm with the counterparts in [13].

\section{CONCLUSIONS}

In this paper, we have derived exact expressions of outage probability, average SER, and average sum-rate for twoway AF relaying networks under i.n.i.d. Nakagami- $m$ fading channels with integer and integer plus one-half values of fading parameters. We have shown that our results reduce to the previously published work for Rayleigh fading channels as special cases. Our analysis has been verified by simulation.

\section{APPENDIX}

\section{A. Proof of Theorem 1}

By using (8), the CDF of $\gamma_{T_{i}}, F_{\gamma_{T_{i}}}(\gamma)$ can be expressed as

$$
F_{\gamma_{T_{i}}}(\gamma)=\int_{0}^{\infty} \operatorname{Pr}\left[\frac{\gamma_{i} \gamma_{j}}{2 \gamma_{i}+\gamma_{j}} \leq \gamma \mid \gamma_{i}\right] f_{\gamma_{i}}\left(\gamma_{i}\right) d \gamma_{i}
$$

$$
\begin{aligned}
= & \int_{0}^{\gamma} \operatorname{Pr}\left[\gamma_{j} \geq \frac{2 \gamma \gamma_{i}}{\gamma_{i}-\gamma} \mid \gamma_{i}\right] f_{\gamma_{i}}\left(\gamma_{i}\right) d \gamma_{i} \\
& +\int_{\gamma}^{\infty} \operatorname{Pr}\left[\gamma_{j} \leq \frac{2 \gamma \gamma_{i}}{\gamma_{i}-\gamma} \mid \gamma_{i}\right] f_{\gamma_{i}}\left(\gamma_{i}\right) d \gamma_{i} \\
= & I_{1}(\gamma)+I_{2}(\gamma),
\end{aligned}
$$

where

$$
\begin{gathered}
I_{1}(\gamma)=\int_{0}^{\gamma} f_{\gamma_{i}}\left(\gamma_{i}\right) d \gamma_{i} \\
=F_{\gamma_{i}}(\gamma)=1-\frac{\Gamma\left(m_{i},\left(m_{i} / \bar{\gamma}_{i}\right) \gamma\right)}{\Gamma\left(m_{i}\right)}, \\
I_{2}(\gamma)=\int_{\gamma}^{\infty} F_{\gamma_{j}}\left(\frac{2 \gamma \gamma_{i}}{\gamma_{i}-\gamma}\right) f_{\gamma_{i}}\left(\gamma_{i}\right) d \gamma_{i} \\
=\frac{\Gamma\left(m_{i},\left(m_{i} / \bar{\gamma}_{i}\right) \gamma\right)}{\Gamma\left(m_{i}\right)}-\frac{m_{i}^{m_{i}}}{\bar{\gamma}_{i}^{m_{i}} \Gamma\left(m_{i}\right) \Gamma\left(m_{j}\right)} I_{3}(\gamma),
\end{gathered}
$$

and

$$
I_{3}(\gamma)=\int_{\gamma}^{\infty} \gamma_{i}^{m_{i}-1} e^{-\frac{m_{i} \gamma_{i}}{\gamma_{i}}} \Gamma\left(m_{j}, \frac{2 \gamma_{i}}{\gamma_{i}-\gamma} \frac{m_{j} \gamma}{\bar{\gamma}_{j}}\right) d \gamma_{i} .
$$

The integral $I_{3}(\gamma)$ in (25) can be written in its general form as

$$
I_{3}(\gamma)=\int_{y}^{\infty} x^{a-1} e^{-b x} \Gamma\left(c, 2 \ell+\frac{2 \ell y}{x-y}\right) d x
$$

where $a, c$ are positive integers and $b, \ell$ are real numbers. By using [14, Eq. (8.352.2)] and [14, Eq. (1.111)], the incomplete gamma function of the integral in (26) can be expressed as

$$
\begin{aligned}
\Gamma\left(c, 2 \ell+\frac{2 \ell y}{x-y}\right) & =(c-1) ! \exp \left(-2 \ell-\frac{2 \ell y}{x-y}\right) \\
& \times \sum_{k=0}^{c-1} \sum_{v=0}^{k} \frac{(2 \ell)^{k}}{k !}\left(\begin{array}{l}
k \\
v
\end{array}\right)\left(\frac{y}{x-y}\right)^{v} .
\end{aligned}
$$

Using (26) and (27), after a simple transformation of the variables, $I_{3}(\gamma)$ can be given by

$$
\begin{aligned}
& I_{3}(\gamma)=(c-1) ! e^{-2 \ell} \sum_{k=0}^{c-1} \sum_{v=0}^{k} \frac{(2 \ell)^{k}}{k !}\left(\begin{array}{l}
k \\
v
\end{array}\right) \\
& \times \int_{y}^{\infty} x^{a-1} \exp \left(-b x-\frac{2 \ell y}{x-y}\right)\left(\frac{y}{x-y}\right)^{v} d x .
\end{aligned}
$$

The inner integral of (28) can be solved by using [14, Eq. (3.471.9)], resulting in

$$
\begin{aligned}
I_{3}(\gamma) & =(c-1) ! e^{-2 \ell-b y} \sum_{k=0}^{c-1} \sum_{v=0}^{k} \sum_{r=0}^{a-1} \frac{(2 \ell)^{k}}{k !}\left(\begin{array}{c}
k \\
v
\end{array}\right)\left(\begin{array}{c}
a-1 \\
r
\end{array}\right) \\
& \times y^{a-r+v-1} 2\left(\frac{2 \ell y}{b}\right)^{\frac{r-v+1}{2}} K_{v-r-1}(2 \sqrt{2 \ell y b}) .
\end{aligned}
$$

With the help of (22), (23), (24) and (29), we get (4).

\section{B. Proof of Theorem 2}

We can rewrite (1) as follows:

$$
\gamma_{T_{i}}=\frac{1}{X_{i}+X_{j}}
$$


where $X_{i}=1 / \gamma_{i}, X_{j}=2 / \gamma_{j}$.

$$
\Phi_{X_{i}}(s)=\mathbb{E}_{X_{i}}\left\{\exp \left(-s X_{i}\right)\right\}=\int_{0}^{\infty} e^{-\frac{s}{\gamma}} f_{\gamma_{i}}(\gamma) d \gamma .
$$

Substituting (2) into (31) and using [14, Eq.(3.471.9)], we get

$$
\Phi_{X_{i}}(s)=\frac{2}{\Gamma\left(m_{i}\right)}\left(\frac{m_{i} s}{\bar{\gamma}_{i}}\right)^{\frac{m_{i}}{2}} K_{m_{i}}\left(2 \sqrt{\frac{m_{i} s}{\bar{\gamma}_{i}}}\right) .
$$

Similarly, we get

$$
\Phi_{X_{j}}(s)=\frac{2}{\Gamma\left(m_{j}\right)}\left(\frac{2 m_{j} s}{\bar{\gamma}_{j}}\right)^{\frac{m_{j}}{2}} K_{m_{j}}\left(2 \sqrt{\frac{2 m_{j} s}{\bar{\gamma}_{j}}}\right) .
$$

Let us define $Y_{i}=X_{i}+X_{j}$. Since $X_{i}$ and $X_{j}$ are statistically independent, by using (32) and (33), the MGF of $Y_{i}$ can be expressed as

$$
\begin{aligned}
\Phi_{Y_{i}}(s)= & \Phi_{X_{i}}(s) \Phi_{X_{j}}(s) \\
= & \frac{4}{\Gamma\left(m_{i}\right) \Gamma\left(m_{j}\right)}\left(\frac{m_{i} s}{\bar{\gamma}_{i}}\right)^{\frac{m_{i}}{2}}\left(\frac{2 m_{j} s}{\bar{\gamma}_{j}}\right)^{\frac{m_{j}}{2}} \\
& \times K_{m_{i}}\left(2 \sqrt{\frac{m_{i} s}{\bar{\gamma}_{i}}}\right) K_{m_{j}}\left(2 \sqrt{\frac{2 m_{j} s}{\bar{\gamma}_{j}}}\right) .
\end{aligned}
$$

Then, the CDF of $\gamma_{T_{i}}, F_{\gamma_{T_{i}}}(\gamma)$, can be shown as

$$
F_{\gamma_{T_{i}}}(\gamma)=1-F_{Y_{i}}(1 / \gamma)=1-\left.\mathcal{L}^{-1}\left(\Phi_{Y_{i}}(s) / s\right)\right|_{1 / \gamma},
$$

where $\mathcal{L}^{-1}(\cdot)$ stands for the inverse Laplace transform. Based on (34) and (35) and using [14, Eq.(8.468)], [16, vol.5, Eq.(2.2.1.11)], we obtain (5).

\section{Proof of Corollary 1}

Utilizing the fact that the PDF of $Y_{i}$ is $f_{Y_{i}}(\gamma)=$ $\mathcal{L}^{-1}\left\{\Phi_{Y_{i}}(s)\right\}$. Next, applying [16, vol.5, Eq.(2.2.1.11)], [14, Eq.(8.950.2)] and the identity $f_{\gamma_{T_{i}}}(\gamma)=\gamma^{-2} f_{Y_{i}}(1 / \gamma)$, we get (7), which completes the proof.

D. Calculation of the Integral $J=$ $\int_{0}^{\infty} x^{m} e^{-p x} \ln (1+x) K_{n}(q x) d x$

Using [16, vol. 3, Eq. (8.4.23.3)], [16, vol. 3, Eq. (8.4.6.5)], [16, vol. 3, Eq. (8.3.2.21)] and after some elementary manipulations, we can write $J$ as

$$
\begin{aligned}
J=\sqrt{\pi} \int_{0}^{\infty} & \left\{x^{m} e^{(q-p) x} H_{2,2}^{1,2}\left[x \mid \begin{array}{cc}
(1,1) & (1,1) \\
(1,1) & (0,1)
\end{array}\right]\right. \\
& \left.\times H_{1,2}^{2,0}\left[2 q x \mid \begin{array}{c}
\left(\frac{1}{2}, 1\right) \\
(n, 1),(-n, 1)
\end{array}\right]\right\} d x,
\end{aligned}
$$

where $H_{C, D}^{A, B}[\cdot]$ is the Fox's $H$-function [16, vol. 3, Eq. (8.3.1.1)].

From [17, Eq. (2.6.2)], we get

$$
\begin{aligned}
& J=\sqrt{\pi}(p-q)^{-m-1} \\
& \times H_{1,[2: 1], 0,[2: 2]}^{1,2,0,1,2}\left[\begin{array}{c|c}
\frac{1}{p-q} & (2,1) \\
& (1,1),(1,1) ;(0.5,1) \\
\frac{2 q}{p-q} & (1,1),(0,1) ;(n, 1),(-n, 1)
\end{array}\right],
\end{aligned}
$$

where $H_{E,[A: C], F,[B: D]}^{K, N, N^{\prime}, M, M^{\prime}}[\cdot]$ is the generalized Fox's $H$-function [17, Eq. (2.2.1)].

\section{REFERENCES}

[1] B. Rankov and A. Wittneben, "Spectral efficient protocols for halfduplex fading relay channels," IEEE J. Sel. Areas Commun., vol. 25, no. 2, pp. 379-389, Feb. 2007.

[2] P. Larsson and N. Johansson, "Interference cancellation in wireless relaying networks," United States Patent no. 7.336.930, Apr. 2004.

[3] J. N. Laneman, D. Tse, and G. Wornell, "Cooperative diversity in wireless networks: Efficient protocols and outage behavior," IEEE Trans. Inf. Theory, vol. 50, no. 12, pp. 3062-3080, Dec. 2004.

[4] R. U. Nabar, H. Bölcskei, and F. W. Kneubuhler, "Fading relay channels: Performance limits and space-time signal design," IEEE J. Sel. Areas Commun., vol. 22, no. 6, pp. 1099-1109, Aug. 2004.

[5] T. A. Tsiftsis, G. K. Karagiannidis, P. T. Mathiopoulos, and S. A. Kotsopoulos, "Nonregenerative dual-hop cooperative links with selection diversity," EURASIP J. Wireless Commmun. Netw., vol. 2006, pp. 1-8, May 2006.

[6] H. A. Suraweera, R. H. Y. Louie, Y. H. Li, G. K. Karagiannidis, and B. Vucetic, "Two hop amplify-and-forward transmission in mixed Rayleigh and Rician fading channels," IEEE Commun. Lett., vol. 13, no. 4, pp. 227-229, 2009.

[7] X. Lei, P. Fan, L. Fan, K. K. Wong, E. G. Larsson, and T. Q. Duong, "Closed-form performance analysis of multihop amplify-and-forward transmission in Nakagami- $m$ fading channels," to be submitted to IEEE Signal Process. Lett., 2010.

[8] N. Yang, M. Elkashlan, and J. Yuan, "Outage probability of multiuser relay networks in Nakagami- $m$ fading channels," IEEE Trans. Veh. Technol., vol. 59, no. 5, pp. 2120-2132, June 2010.

[9] J. Yang, P. Fan, Q. C. Chen, and X. Lei, "Delay-limited throughput analysis of dual-hop amplify-and-forward HARQ transmissions over asymmetric fading channels," Submitted to IEEE Trans. Veh. Tech.

[10] Y. Han, S. H. Ting, C. K. Ho, and W. H. Chin, "Performance bounds for two-way amplify-and-forward relaying," IEEE Trans. Wireless Commun., vol. 8, no. 1, pp. 432-439, Jan. 2009.

[11] J. Ping and S. H. Ting, "Rate performance of AF two-way relaying in low SNR region," IEEE Commun. Lett., vol. 13, no. 4, pp. 233-235, Apr. 2009.

[12] Q. Li, S. H. Ting, A. Pandharipande, and Y. Han, "Adaptive two-way relaying and outage analysis," IEEE Trans. Wireless Commun., vol. 8, no. 6, pp. 3288-3299, June 2009.

[13] T. Q. Duong, L.-N. Hoang, and V. N. Q. Bao, "On the performance of two-way amplify-and-forward relay," IEICE Trans. Commun., vol. E92-B, no. 12, pp. 3957-3959, Dec. 2009.

[14] I. S. Gradshteyn and I. M. Ryzhik, Table of Integrals, Series, and Products, 6th ed. San Diego, CA: Academic Press, 2000.

[15] M. K. Simon and M. S. Alouini, Digital Communication Over Fading Channels: A Unified Approach to Performance Analysis, 6th ed. NY: Wiley-Interscience, 2000.

[16] A. P. Prudnikov, Y. A. Brychkov, and O. I. Marichev, Integrals and Series. NY: Gordon and Breach Science, 1986, vol. 3,5.

[17] A. M. Mathai and R. K. Saxena, The H-function with Applications in Statistics and Other Disciplines. NY: Wiley, 1978.

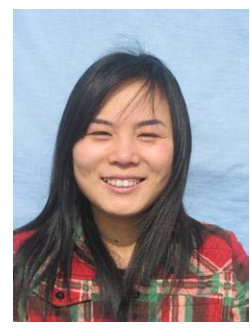

Jing Yang received the B.S. degree in communications engineering from Sichuan Normal University, Chengdu, China, in 2006. She is currently working toward her Ph.D degree at the Institute of Mobile Communications, Southwest Jiaotong University, Chengdu, China. Her research interests include the cross-layer optimization and cooperative diversity. 


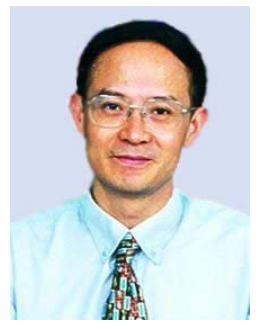

Pingzhi Fan (M'93-SM'99) received his M.S. degree in Computer Science from the Southwest Jiaotong University, PRC, in 1987, and Ph.D. degree in Electronic Engineering from the Hull University, U.K., in 1994. He is currently a professor and director of the institute of mobile communications, Southwest Jiaotong University, PRC, and a guest professor of Leeds University, UK (1997-), a guest professor Shanghai Jiaotong University (1999-). He was a recipient of the UK ORS Award (1992), and the NSFC Outstanding Young Scientist Award (1998). He is the inventor of 22 patents, and the author of over 350 research papers and 8 books, including six books published by John Wiley \& Sons Ltd, RSP (1996), IEEE Press $(2003,2006)$ and Springer (2004) and Nova Science (2007), respectively. His research interests include spread-spectrum and CDMA technology, information theory \& coding, sequence design \& applications, radio resource management, etc.

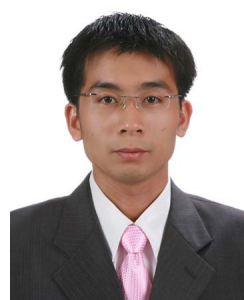

Trung Q. Duong was born in HoiAn town, Quang Nam province, Vietnam, in 1979. He received the B.S. degree in electrical engineering from $\mathrm{Ho}$ Chi Minh City University of Technology, Vietnam in 2002, and the M.Sc. degree in computer engineering from Kyung Hee University, South Korea, in 2005. In April 2004, he joined the faculty of electrical engineering of Ho Chi Minh City University of Transport, Vietnam. He was a recipient of the Korean Government IT Scholarship Program for International Graduate Students from 2003 to 2007. He was awarded the Best Paper Award of IEEE Student Paper Contest IEEE Seoul Section in December 2006 and was listed in the finalist for the best paper award at the IEEE Radio and Wireless Symposium, San Diego, U.S.A. in 2009. He has been acting as an active reviewer for numerous journals/conferences and a TPC member for more than 10 conferences. He has been a TPC co-chair of the International Workshop on Multimedia Streaming (MultiStreams2011) and a visiting scholar at Polytechnic Institute of New York University from December 2009 to January 2010. In December 2007, he joined the Radio Communication Group, Blekinge Institute of Technology, Sweden as a research staff working toward his Ph.D. degree in radio communications. He is the author or coauthor of some 50 technical papers in the area of wireless and mobile communications. His current research interests include cross-layer optimization, cooperative communications, cognitive radio networks, and applied game theory in wireless systems.

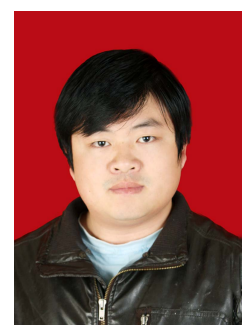

Xianfu Lei is with Institute of Mobile Communications (IMC), Key Lab of Information Coding and Transmission, Southwest Jiaotong University, China. $\mathrm{He}$ is serving as the treasurer of IEEE Information Theory Society Chengdu Chapter. He served as workshop co-chair of PerMedia'10. He serves as publication chair for IWSDA'11 and TPC member for Globecom'11, ICC'11, WCNC'11, VTC'11, etc. His research interests include digital communications over fading channels, relay-assisted and cooperative communications, cognitive communications,

wireless communication theory, etc. 